\title{
Rastreamento do Câncer do Colo do Útero em Mulheres de 25 a 64 anos: Indicadores do Primeiro Exame Citopatológico Informado no Siscolo, 2007-2013
}

ttps://doi.org/10.32635/2176-9745.RBC.2022v68n1.1520

\author{
Cervical Cancer Screening in Women Aged 25 to 64 Years: Indicators of the First Cytopathological Examination Registered \\ at Siscolo, 2007-2013 \\ Tamizaje Masivo de Cáncer de Cuello Uterino en Mujeres de 25 a 64 años: Indicadores de la Primera Vez de Examen \\ Citopatológico en Siscolo, 2007-2013
}

\author{
Maria Beatriz Kneipp Dias'; Luciana Leite de Mattos Alcântara²; Vania Reis Girianelli³; Arn Migowski; Caroline Madalena Ribeiro5; \\ Jeane Tomazelli6
}

\section{RESUMO}

Introduçáo: $\mathrm{O}$ câncer do colo do útero apresenta elevadas taxas de incidência e mortalidade em mulheres no Brasil. O monitoramento das açóes de controle tem sido limitado ao número de exames realizados e não às mulheres examinadas em razão da falta de um identificador único no sistema de informação. Objetivo: Analisar indicadores das açôes de rastreamento do câncer do colo do útero entre mulheres de 25-64 anos com registro do primeiro exame citopatológico informado no Siscolo no período de 2007 a 2013. Método: Estudo de séries temporais dos indicadores das ações de rastreamento do câncer do colo do útero, calculados para mulheres de 25-64 anos que tiveram o primeiro exame citopatológico identificado no Siscolo entre 2007 e 2013. A tendência temporal foi avaliada usando a regressão linear generalizada pelo método de Prais-Winsten. Resultado: $\mathrm{O}$ indicador de captação mostrou tendência crescente apenas na Região Sul ( $\mathrm{p}=0,038)$, enquanto a positividade geral (qualquer alteraçáo citopatológica) manteve-se estacionária no país $(\mathrm{p} \geq 0,108)$. A representatividade da zona de transformaçáo apresentou tendência decrescente em todas as Regiōes $(\mathrm{p}<0,05)$, enquanto a razão entre lesão intraepitelial de alto grau e carcinoma epidermoide apontou tendência crescente nas Regiôes Nordeste (10,3 em 2007 para 13,3 em 2013), Sudeste (17,5 para 20,2) e Sul (20,2 para 30,4). Conclusáo: A razão entre lesão de alto grau e câncer crescente em três Regióes do país indica bom desempenho do rastreamento nessas localidades. Indicadores com tendência estacionária e tendência decrescente da representatividade da zona de transformaçáo necessitam de acompanhamento.

Palavras-chave: sistema de informação em saúde; neoplasias do colo do útero; teste de papanicolaou; programas de rastreamento; estudos de séries temporais.

\section{ABSTRACT}

Introduction: Cervical cancer has high rates of incidence and mortality in women in Brazil. Monitoring of control actions has been limited to the number of tests performed and not to the women examined due to the lack of a unique identifier in the information system. Objective: To analyze indicators of cervical cancer screening actions among women aged 25-64 years with a record of the first cytopathological examination reported at Siscolo from 2007 to 2013. Method: Study of time series of indicators of cervical cancer screening actions were calculated for women aged 25-64 years who had their first cytopathological exam identified at Siscolo between 2007 and 2013. Trend analysis was performed using Prais-Winsten regression. Result: The uptake indicator showed an increasing trend only in the South $(\mathrm{p}=0.038)$ while overall positivity (any cytopathological alteration) remained stationary in the country $(\mathrm{p} \geq 0.108)$. Transformation zone sampling rate showed a decreasing trend in all regions $(p<0.05)$, while the ratio between high-grade intraepithelial lesion and squamous cell carcinoma showed an increasing trend in the Northeast (10.3 in 2007 to 13.3 in 2013), Southeast (17.5 to 20.2) and South (20.2 to 30.4) regions. Conclusion: The increasing ratio between high-grade lesion and cancer in three regions of the country indicates a good screening performance in these locations. Indicators with a stationary trend and a decreasing trend in the transformation zone sampling rate need monitoring.

Key words: health information systems; uterine cervical neoplasms; papanicolaou test; mass screening; time series studies.

\section{RESUMEN}

Introducción: El cáncer de cuello uterino tiene altas tasas de incidencia y mortalidad en mujeres en Brasil. El seguimiento de las acciones de control se ha limitado al número de pruebas realizadas y no mujeres examinadas debido a falta de un identificador único en sistema de información. Objetivo: Analizar indicadores de acciones de tamizaje de cáncer cervicouterino en mujeres de 25-64 años con registro del primer examen citopatológico reportado en Siscolo en período 2007 a 2013. Método: Estudio de series temporales de indicadores de acciones de tamizaje masivo del cuello uterino calculado para mujeres de 25-64 años a las que se les identificó su primer examen citopatológico en Siscolo entre 2007 y 2013. La tendencia temporal se evaluó mediante regresión lineal generalizada mediante el método PraisWinsten. Resultado: El indicador de captación mostró tendencia creciente solo en Sur $(\mathrm{p}=0,038)$ mientras que la positividad general (cualquier alteración citopatológica) se mantuvo estacionaria en el país $(\mathrm{p} \geq 0,108)$. La representatividad de la zona de transformación mostró una tendencia decreciente en todas regiones $(\mathrm{p}<0,05)$, mientras que la relación entre lesiones intraepitelial de alto grado y carcinoma células escamosas mostró una tendencia creciente en regiones Noreste (10,3 en 2007 a 13,3 en 2013), Sudeste $(17,5$ a 20,2$)$ y Sur $(20,2$ a 30,4). Conclusión: La relación entre lesiones de alto grado y aumento de cáncer en tres regiones indica buen desempeńo de detección en estos lugares. Los indicadores con tendencia estacionaria y tendencia decreciente en representatividad de la zona de transformación necesitan monitoreo.

Palabras clave: sistemas de información en salud; neoplasias del cuello uterino; prueba de papanicolaou; tamizaje masivo; estudios de series temporales.

\footnotetext{
1,4,5,6Instituto Nacional de Câncer José Alencar Gomes da Silva (INCA). Rio de Janeiro (RJ), Brasil. E-mails: mdias@inca.gov.br; arn@inca.gov.br; cribeiro@inca.gov.br; jtomazelli@inca.gov.br. Orcid iD: https://orcid.org/0000-0002-5847-9830; Orcid iD: https://orcid.org/0000-0002-4861-2319; Orcid iD: https://orcid.org/00000003-2690-5791; Orcid iD: https://orcid.org/0000-0002-2472-3444

${ }^{2}$ Universidade Federal do Rio de Janeiro (UFRJ), Instituto Alberto Luiz Coimbra de Pós-Graduação e Pesquisa em Engenharia (Coppe). Rio de Janeiro (RJ), Brasil. E-mail: lucianaleite@peb.ufrj.br. Orcid iD: https://orcid.org/0000-0003-2309-6976

${ }^{3}$ Fundação Oswaldo Cruz (Fiocruz), Escola Nacional de Saúde Pública Sérgio Arouca (Ensp). Rio de Janeiro (RJ), Brasil. E-mail: vaniagirianelli@yahoo.com.br. Orcid iD: https://orcid.org/0000-0002-8690-9893

Endereço para Correspondência: Jeane Tomazelli. Rua Marquês de Pombal, 125, 70 andar - Centro. Rio de Janeiro (RJ), Brasil. CEP $22230-240$. E-mail: jtomazelli@inca.gov.br
} 


\section{INTRODUÇÃO}

Apesar de ser uma doença evitável, o câncer do colo do útero ainda apresenta altas taxas de incidência e mortalidade em mulheres, principalmente nos países em desenvolvimento. A doença é precedida por lesões pré-malignas que, se detectadas e tratadas oportunamente, não evoluem para câncer. Estudos sobre a história natural da doença estimam que o intervalo de tempo da progressão de alterações celulares pela infecção persistente pelo Papilomavírus Humano (HPV) e a neoplasia maligna está em torno de 10 a 15 anos $^{1}$.

O exame citopatológico de Papanicolaou é a técnica adotada no Brasil para o rastreamento do câncer do colo do útero. As Diretrizes Brasileiras para o Rastreamento do Câncer do Colo do Útero recomendam que o exame seja realizado a cada três anos, após dois exames anuais consecutivos sem alteraçôes². A Organização Mundial da Saúde (OMS) recomenda uma cobertura de pelo menos $70 \%$ da população-alvo para que haja redução na morbimortalidade do câncer do colo do útero ${ }^{3}$.

No Brasil, açóes de controle do câncer do colo do útero foram iniciadas com a implantaçáo do Programa Atenção Integral à Saúde da Mulher (Paism) na década de 1980. Na década seguinte, em 1997, teve início o projeto Viva Mulher para organização do Programa Nacional de Controle do Câncer do Colo Uterino (PNCCCU) ${ }^{2}$. Para o monitoramento das açóes, foi desenvolvido o Sistema de Informação do Controle do Câncer do Colo do Útero $(\text { Siscolo })^{4}$, que começou a ser utilizado nacionalmente em 1999.

O Siscolo registra os exames citopatológicos e histopatológicos realizados no Sistema Único de Saúde (SUS). As informaçôes são enviadas por todas as Unidades Federativas ao Departamento de Informática do SUS (DATASUS), que as consolida compondo o sistema nacional. Ele também contempla um conjunto de informaçôes que são importantes para a gestão do programa de rastreamento do câncer do colo do útero. Entretanto, apresenta limitaçóes como: registro de exames e não de mulheres, processo de consolidação de informaçóes nacional dependente da rotina de envio e monitoramento de suas bases de dados, realizado pelos entes federativos estaduais e municipais ${ }^{4}$. Diante desses problemas, fica comprometido o cálculo de indicadores usados em programas de rastreamento de câncer, e a comparabilidade com outros países, que são essenciais para monitorar sua efetividade ${ }^{5-9}$. Os estudos nacionais acabam se atendo no máximo a indicadores proxy que possuem importantes limitaçóes ${ }^{10-13}$. Uma alternativa é utilizar estratégias de relacionamento probabilístico entre bases de dados para identificar as mulheres e o seu seguimento, visando a uma maior aproximação da realidade ${ }^{14-16}$.

Este artigo tem por objetivo analisar indicadores das açôes de rastreamento do câncer do colo do útero entre mulheres de 25 a 64 anos com registro do primeiro exame citopatológico informado no Siscolo, no período de 2007 a 2013, bem como sua evolução temporal.

\section{MÉTODO}

Trata-se de um estudo de séries temporais dos indicadores referentes às açôes de rastreamento do câncer do colo do útero no Brasil, calculados a partir dos registros das mulheres de 25 a 64 anos de idade que tiveram o primeiro exame citopatológico identificado na base do Siscolo segundo Regiôes do país entre 2007 e 2013. O período do estudo foi definido com base no ano de implantaçáo da versão do Siscolo com a nomenclatura citopatológica atualizada (2007) até o ano de sua descontinuidade (2013).

Os dados identificados foram acessados na base nacional do Siscolo, o qual não possui uma variável que possa ser utilizada como chave de identificação das mulheres. Para tanto, foi realizado relacionamento probabilístico entre as bases de dados contendo os exames registrados no período para identificar o primeiro exame registrado para cada mulher.

O relacionamento probabilístico foi realizado utilizando-se o programa $\mathrm{R}^{17}$, versão 3.5.3, e os pacotes RecordLinkage e SoundexBR, tendo como chaves de blocagem nome da mulher, nome da máe e a data de nascimento. A data de coleta do exame foi utilizada como critério para identificar o primeiro exame da mulher.

Na Figura 1, apresenta-se o fluxo de identificação das mulheres com primeiro exame registrado no Siscolo a cada ano, no período do estudo. Os exames de seguimento não foram incluídos no escopo deste estudo.

Diversos indicadores são utilizados nos programas organizados de rastreamento, a fim de monitorar e avaliar o desempenho das açôes, ${ }^{5,9}$. Neste estudo, foram analisados os indicadores: proporção de mulheres captadas, percentual de positividade, percentual de representatividade da zona de transformação $(Z T)$ e razão entre lesão de alto grau e câncer.

O indicador de captaçáo possibilita mensurar, entre as mulheres que realizam o exame, aquelas que o fazem pela primeira vez na vida. Apesar de o banco analisado apresentar as mulheres de 25 a 64 anos que foram registradas pela primeira vez no período, para o cálculo de 'mulheres captadas', foi utilizada a informação disponível na variável "fez preventivo anterior", uma vez que as mulheres poderiam ter feito exame em idade mais jovem ou em serviços náo credenciados ao SUS. 


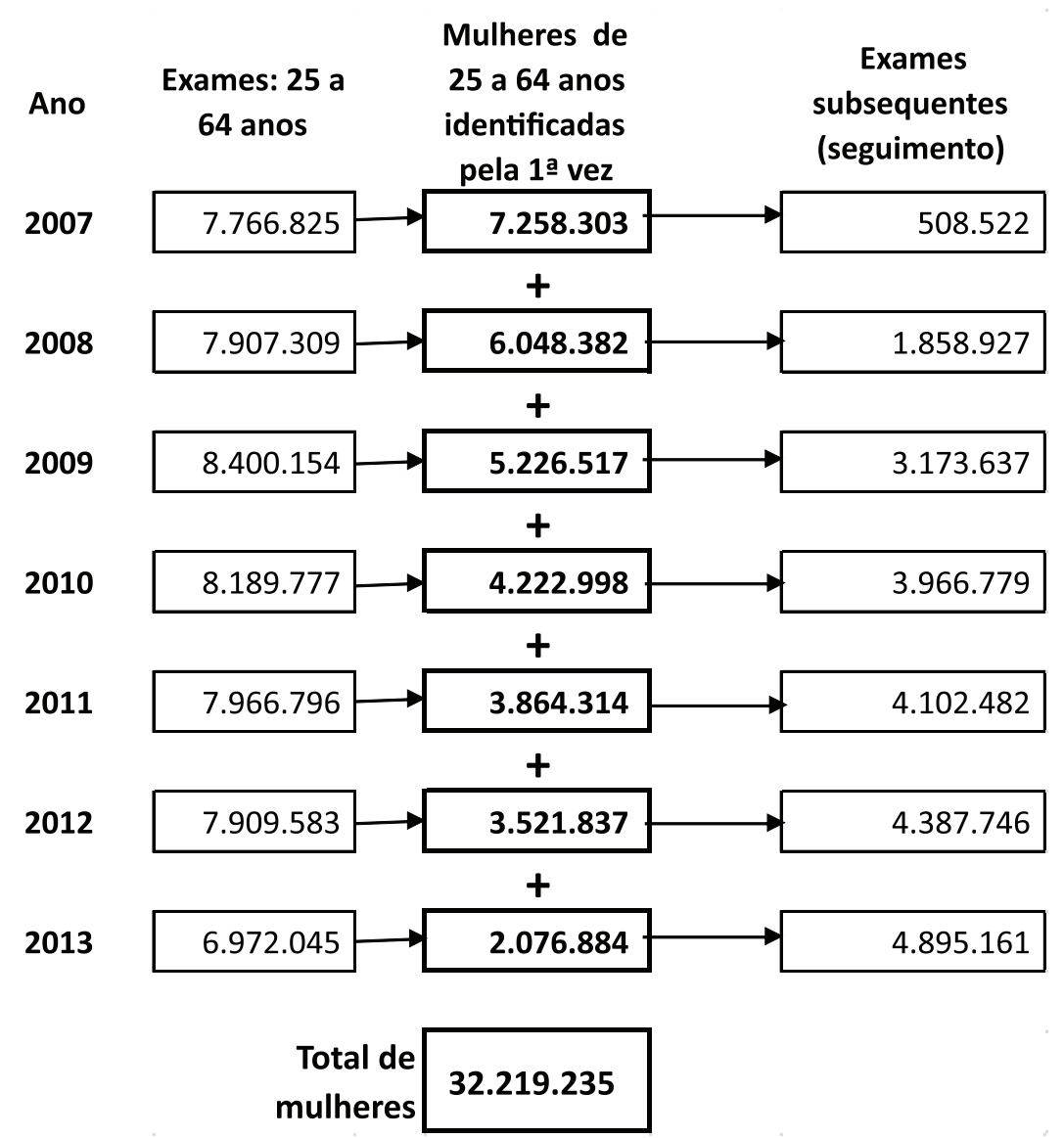

Figura 1. Fluxo de identificação de mulheres pelo relacionamento probabilístico dos exames citopatológicos por ano. Brasil, 2007-2013

Fórmula: Número de mulheres examinadas pela primeira vez (variável preventivo anterior $=$ não) na população-alvo/número de mulheres na população-alvo examinadas.

O percentual de positividade avalia quantas mulheres tiveram resultado citopatológico alterado em relação à população rastreada ${ }^{12}$. Esse indicador, quando restrito às mulheres que realizam o exame pela primeira vez, expressa os exames alterados em mulheres que nunca haviam realizado exame citopatológico anteriormente. São consideradas mulheres com exames alterados aquelas que apresentaram pelo menos um dos seguintes resultados: células escamosas atípicas de significado indeterminado (ASC-US e ASC-H); lesão intraepitelial de baixo grau (LSIL); lesão intraepitelial de alto grau (HSIL); carcinoma epidermoide invasor (CEI); células glandulares atípicas de significado indeterminado (AGC); adenocarcinoma in situ (AIS) e invasor, células atípicas de origem indefinida e outras neoplasias ${ }^{2}$. Esse indicador foi calculado para o conjunto de mulheres de 25 a 64 anos (positividade geral) e para aquelas que informaram não ter realizado exame preventivo anteriormente (positividade em mulheres captadas). a. Percentual (ou Índice) de positividade nas mulheres examinadas no período

Fórmula: Número de mulheres da populaçãoalvo com resultado de exame alterado/número de mulheres examinadas na população-alvo, ano e Região, multiplicado por 100 .

b. Percentual (ou Índice) de positividade em mulheres da população-alvo captadas

Fórmula: Número de mulheres da população-alvo captadas com resultado de exame alterado/número de mulheres captadas na população-alvo, ano e Região, multiplicado por 100 .

A proporção de mulheres com exame citopatológico com representatividade da $\mathrm{ZT}$ é um indicador que permite, principalmente quando avaliado conjuntamente com a taxa de positividade, qualificar a discussão dos resultados alterados, pois a presença de epitélio na ZT aumenta a chance de identificar lesóes ${ }^{18}$. Exames com representatividade da $\mathrm{ZT}$ são os que apresentam no material colhido, além do epitélio escamoso, o epitélio 
metaplásico e/ou glandular, e, portanto, também refletem a qualidade da coleta do material para o exame $e^{2,18}$.

Fórmula: Número de mulheres da população-alvo com exames com representatividade da ZT, dividido pelo número de mulheres da populaçáo-alvo examinadas, multiplicado por 100 .

O indicador razão entre lesão intraepitelial de alto grau e carcinoma epidermoide permite avaliar se o programa está sendo capaz de identificar mais lesôes precursoras do que casos de câncer, entre as atipias escamosas diagnosticadas, indicando efetividade do rastreamento ${ }^{19}$.

Fórmula: Número de mulheres na população-alvo, com resultado citopatológico de lesão intraepitelial de alto grau dividido pelo número de mulheres na população-alvo com resultado citopatológico de carcinoma epidermoide.

Realizou-se regressão linear generalizada pelo método de Prais-Winsten para analisar a tendência, e a estatística de Durbin-Watson para avaliar a autocorrelação serial; ou seja, a dependência de uma medida com seus próprios valores anteriores da série ${ }^{20,21}$. Quando a tendência foi estatisticamente significativa e a estatística de Durbin-Watson ficou na área de indecisão, esta foi classificada como indeterminada. Foi calculada a variação média anual (do inglês, Annual Percent Change - APC) dos indicadores para as Regióes com intervalo de confiança de 95\% $\left(\mathrm{IC}_{95 \%}\right)$. Adicionalmente, foi calculada a correlaçáo de Pearson entre o indicador de positividade geral e de representatividade da ZT, e classificada conforme proposto por Callegari-Jacques ${ }^{22}: r=0$, nula; $0<r \leq|0,3|$, fraca; $|0,3|<\mathrm{r} \leq|0,6|$, moderada; $|0,6|<\mathrm{r} \leq|0,9|$, forte; $|0,9|$ $<\mathrm{r}<|1|$, muito forte; e $\mathrm{r}=1$, classificada como perfeita. Para todas as análises estatísticas, foi considerado o nível de significância de $5 \%$.
Esta pesquisa faz parte de um projeto aprovado pelo Comitê de Ética em Pesquisa (CEP) do Instituto Nacional de Câncer José Alencar Gomes da Silva (INCA) sob o número CAAE: 68203117.1.0000.5274.

\section{RESULTADOS}

No período de 2007 a 2013, foram identificados no Siscolo 71.527 .653 exames citopatológicos de rastreamento satisfatórios, dos quais, 55.112 .489 (77\%) na população-alvo de 25 a 64 anos. O estudo identificou 32,3 milhóes de mulheres, na faixa etária alvo, resultando em uma razão de 1,71 exames/mulher. A região Norte apresentou menor razão de exames por mulheres examinadas $(1,59)$, enquanto as Regiōes Sudeste e Centro-Oeste apresentaram razão de exames de 1,82 (Tabela 1).

Ao longo dos anos, houve redução gradativa do número de mulheres identificadas pela primeira vez no Siscolo. Entre 2007 e 2010, a redução foi de aproximadamente mil mulheres/ano, com maior percentual, de 19\%, entre 2010 e 2009, diminuindo nos anos seguintes (menos de 9\%). Entretanto, no ano de 2013, a redução foi de aproximadamente $41 \%$, correspondendo a $1,4 \mathrm{mil}$ mulheres a menos em relação ao ano anterior.

A Tabela 2 mostra a tendência dos indicadores analisados no período. $\mathrm{O}$ indicador de captação apresentou tendência crescente apenas na Regiāo Sul $(\mathrm{p}=0,038)$, com incremento anual de $6,83\left(\mathrm{IC}_{95 \%} 0,83 ; 13,48\right)$, variando de 6,1 em 2007 a 7,8 em 2013.

A proporçáo de positividade geral apontou tendência estacionária em todas as Regióes ( $\mathrm{p} \geq 0,108)$. A proporção de positividade em mulheres captadas, no entanto, apresentou incremento anual de 4,10 ( $\mathrm{IC}_{95 \%}-0,18$; $8,56)$ na Região Centro-Oeste, embora com significância estatística limítrofe $(\mathrm{p}=0,066)$, enquanto as demais Regiōes mantiveram-se estacionárias $(\mathrm{p} \geq 0,327)$. Observou-se, contudo, que a Região Centro-Oeste se destaca com uma proporção de positividade em mulheres captadas

Tabela 1. Distribuição do número de exames citopatológicos do colo do útero e mulheres examinadas entre 25 e 64 anos com registro no Siscolo. Brasil, Regiỗes, 2007-2013

\begin{tabular}{lccc}
\hline \multicolumn{1}{c}{ Regiões } & Mulheres & Exames & $\begin{array}{c}\text { Razão exames/mulheres } \\
\text { examinadas }\end{array}$ \\
\hline Norte & 2.048 .233 & 3.250 .933 & 1,59 \\
Nordeste & 8.953 .714 & 14.391 .609 & 1,61 \\
Sudeste & 13.721 .588 & 24.925 .959 & 1,82 \\
Centro-Oeste & 2.167 .592 & 3.942 .451 & 1,82 \\
Sul & 5.328 .108 & 8.601 .537 & 1,61 \\
\hline Brasil & 32.219 .235 & 55.112 .489 & 1,71 \\
\hline
\end{tabular}


Tabela 2. Indicadores em mulheres de 25 a 64 anos, variação e tendências por Regiões. Brasil, 2007-2013

\begin{tabular}{|c|c|c|c|c|c|c|c|c|c|c|}
\hline \multirow{2}{*}{ Indicadores } & \multirow{2}{*}{2007} & \multirow{2}{*}{2008} & \multirow{2}{*}{2009} & \multirow{2}{*}{2010} & \multirow{2}{*}{2011} & \multirow{2}{*}{2012} & \multirow{2}{*}{2013} & Variação anual & \multirow{2}{*}{ Valor-p } & \multirow{2}{*}{ Tendência } \\
\hline & & & & & & & & $\%\left(I C_{95 \%}\right)$ & & \\
\hline \multicolumn{11}{|l|}{ Captação } \\
\hline Centro-Oeste & 11,7 & 9,3 & 12,9 & 13,8 & 15,5 & 11,8 & 10,9 & $0,08(-7,24 ; 7,97)$ & 0,981 & Estacionária \\
\hline Norte & 16,8 & 16,7 & 17,7 & 18,7 & 19,1 & 18,8 & 15,6 & $-0,60(-5,37 ; 4,41)$ & 0,778 & Estacionária \\
\hline Nordeste & 12,8 & 12,8 & 12,9 & 14,2 & 14 & 14,1 & 13,2 & $1,11(-0,64 ; 2,89)$ & 0,219 & Estacionária \\
\hline Sudeste & 7,1 & 6,2 & 5,6 & 6,7 & 7,6 & 7,2 & 7,5 & $2,61(-2,03 ; 7,48)$ & 0,231 & Estacionária \\
\hline Sul & 6,1 & 4,7 & 7,9 & 8 & 6,8 & 8,1 & 7,8 & $6,83(0,83 ; 13,18)$ & 0,038 & Crescente \\
\hline \multicolumn{11}{|c|}{ Positividade geral } \\
\hline Centro-0este & 3,2 & 3,1 & 3 & 2,8 & 3 & 3,4 & 3,3 & $0,76(-3,12 ; 4,80)$ & 0,656 & Estacionária \\
\hline Norte & 2,9 & 2,6 & 2,2 & 2,1 & 2,5 & 2,7 & 2,8 & $0,36(-6,92 ; 8,20)$ & 0,912 & Estacionária \\
\hline Nordeste & 2 & 2 & 1,8 & 1,9 & 2 & 1,9 & 2,2 & $0,92(-1,82 ; 3,74)$ & 0,453 & Estacionária \\
\hline Sudeste & 2,9 & 2,8 & 3,1 & 3,4 & 3,2 & 3,1 & 3,3 & $2,10(-0,52 ; 4,79)$ & 0,108 & Estacionária \\
\hline Sul & 1,7 & 2 & 1,7 & 1,9 & 2 & 1,9 & 1,9 & $1,11(-0,42 ; 2,67)$ & 0,136 & Estacionária \\
\hline \multicolumn{11}{|c|}{ Positividade em mulheres captadas } \\
\hline Centro-Oeste & 3,5 & 4,4 & 4,2 & 3,8 & 4 & 4,7 & 5,0 & $4,10(-0,18 ; 8,56)$ & 0,066 & Estacionária \\
\hline Norte & 2,7 & 2,6 & 2,5 & 2 & 2,3 & 2,5 & 3,1 & $1,46(-6,73 ; 10,37)$ & 0,691 & Estacionária \\
\hline Nordeste & 1,8 & 1,8 & 1,6 & 1,7 & 1,8 & 1,6 & 2,1 & $0,87(-1,31 ; 3,10)$ & 0,377 & Estacionária \\
\hline Sudeste & 2,8 & 2,5 & 2,8 & 3,2 & 3,3 & 3,2 & 2,8 & $1,75(-3,44 ; 7,23)$ & 0,453 & Estacionária \\
\hline Sul & 2,2 & 2,4 & 2,2 & 2,3 & 2,5 & 2,2 & 2,0 & $-1,37(-4,40 ; 1,75)$ & 0,327 & Estacionária \\
\hline \multicolumn{11}{|c|}{ Representatividade da zona de transformação } \\
\hline Centro-0este & 69,3 & 67,9 & 66,5 & 66 & 64,5 & 63,1 & 62,0 & $-1,80(-1,94 ;-1,66)$ & $<0,001$ & Decrescente \\
\hline Norte & 77,5 & 72,7 & 70,5 & 68,5 & 66,2 & 67,6 & 64,0 & $-2,71(-3,61 ;-1,81)$ & $<0,001$ & Decrescente \\
\hline Nordeste & 64,3 & 63,3 & 61,4 & 60,8 & 60 & 59,8 & 55,3 & $-1,67(-2,01 ;-1,33)$ & $<0,001$ & Decrescente \\
\hline Sudeste* & 66,9 & 63,9 & 61,6 & 60,5 & 59,5 & 59,3 & 59,7 & $-1,86(-3,12 ;-0,59)$ & 0,016 & Indeterminada \\
\hline Sul & 67,1 & 65 & 63 & 62,1 & 61,2 & 60,9 & 58,9 & $-1,47(-1,95 ;-0,99)$ & $<0,001$ & Decrescente \\
\hline \multicolumn{11}{|c|}{ Razão alto grau e câncer } \\
\hline Centro-0este & 16,5 & 14,5 & 15,7 & 14,5 & 14,4 & 17,1 & 22,6 & $4,65(-3,15 ; 13,07)$ & 0,21 & Estacionária \\
\hline Norte & 11,2 & 13,8 & 10,2 & 9,7 & 14 & 15,8 & 22,1 & $10,49(-1,81 ; 24,34)$ & 0,094 & Estacionária \\
\hline Nordeste & 10,7 & 10,4 & 11,9 & 11,4 & 11,8 & 11,9 & 13,3 & $3,14(2,00 ; 4,29)$ & 0,001 & Crescente \\
\hline Sudeste & 17,5 & 18,1 & 19,2 & 20,1 & 21,2 & 19,8 & 20,2 & $2,50(0,22 ; 4,83)$ & 0,043 & Crescente \\
\hline Sul & 20,2 & 21,6 & 20,5 & 26,1 & 23,4 & 28,4 & 30,4 & $7,02(5,22 ; 8,86)$ & $<0,001$ & Crescente \\
\hline \multicolumn{11}{|l|}{ Mulheres com } \\
\hline $\begin{array}{l}1^{0} \text { registro de } \\
\text { exame }\end{array}$ & 7.258 .303 & 6.048 .382 & 5.226 .517 & 4.222 .998 & 3.864 .314 & 3.521 .837 & 2.076 .884 & - & - & - \\
\hline
\end{tabular}

Legenda: IC = Intervalo de confiança.

(*) Estatística de Durbin-Watson indicando possível autocorrelação serial.

superior à positividade geral em todo o período, um comportamento similar ocorreu também na Região Sul.

$\mathrm{O}$ indicador de representatividade da ZT apresentou tendência decrescente em todas as Regiōes. A maior redução ocorreu na Região Norte com incremento anual de $-2,71\left(\mathrm{IC}_{95 \%}-3,61 ;-1,81\right)$, variando de 77,5 em 2007 a 64,0 em 2013. Já a menor ocorreu na Região
Sul (APC= $\left.-1,47 ; \mathrm{IC}_{95 \%}-1,95 ;-0,99\right)$, variando de 67,1 em 2007 a 58,9 em 2013. Os valores mais extremos de APC foram nas Regiôes Norte $\left(-2,71, \mathrm{IC}_{95 \%}-3,61 ;-1,81\right)$ e Sudeste $\left(-1,86 ; \mathrm{IC}_{95 \%}-3,12 ;-0,59\right)$. A Região Sudeste, entretanto, apontou possível autocorrelação serial (zona de indecisão) na estatística Durbin-Watson e, portanto, a tendência, embora decrescente e estatisticamente 
significativa ( $\mathrm{p}=0,016)$, foi considerada indeterminada. Todas as demais Regióes tiveram tendência decrescente e significativa $(\mathrm{p}<0,001)$.

O indicador de razão entre lesão de alto grau e câncer apresentou tendência crescente apenas em três Regiôes. Destas, a maior variação percentual ocorreu na Região $\operatorname{Sul}\left(7,02 ; \mathrm{IC}_{95 \%} 5,22 ; 8,86\right)$, variando de $20,3 \mathrm{em} 2007$ a 30,4 em 2013; e a menor na Região Sudeste $\left(2,50 ; \mathrm{IC}_{95 \%}\right.$ 0,22; 4,83), variando de 17,5 em 2007 a 20,2 em 2013.

A Figura 2 apresenta a distribuição dos indicadores de representatividade da ZT e de positividade geral no período de 2007 a 2013, por Região. Observou-se uma correlação negativa entre esses indicadores na maioria das Regióes. A Região Sudeste apresentou correlação negativa forte $(-0,75)$, mas sem significância estatística $(\mathrm{p}=0,055)$. A partir de 2009, a Regiāo Norte mostrou correlação negativa muito forte $(-0,95, \mathrm{p}=0,014)$ e a Região Centro-Oeste, correlação negativa forte $(-0,84)$, mas sem significância estatística $(\mathrm{p}=0,072)$. Apenas a Região Sul apresentou correlação positiva a partir de 2010, mas fraca $(0,21)$ e sem significância estatística $(p=0,790)$.

\section{DISCUSSÃO}

O presente estudo se destaca de outros publicados, por apresentar dados individualizados por mulher e não apenas exames, tendo identificado 55.112.489 exames citopatológicos realizados no Brasil entre 2007 e 2013, em 32.219.235 de mulheres, o que corresponde a uma razão de 1,71 exames por mulher examinada. Esses resultados demonstram as limitaçóes de outros estudos publicados no Brasil até aqui, nos quais exames são considerados como proxy do número de mulheres rastreadas. Além disso, este estudo faz o resgate das coortes rastreadas desde o início dos sistemas de informação no SUS, o que é essencial para avaliação das ações do rastreamento até os dias de hoje por ser uma ação contínua, que demanda seguimento e várias rodadas de rastreamento ao longo de várias décadas da vida das mulheres.

Os indicadores de captação e positividade apresentaram tendência estacionária no período, enquanto a representatividade da ZT, tendência decrescente. Embora os resultados não tenham mostrado significância estatística, merecem ser investigados, uma vez que medidas de ampliação da oferta e qualificação dos exames foram adotadas ao final do período analisado ${ }^{23,24}$.

A pesquisa apresentou o número de mulheres registradas pela primeira vez no Siscolo, sem, contudo, verificar a periodicidade do rastreamento, o que levou a uma diminuição esperada do número de mulheres ao longo do período. $\mathrm{O}$ ano de 2013 com maior reduçáo do número de mulheres reflete o início da implantação do

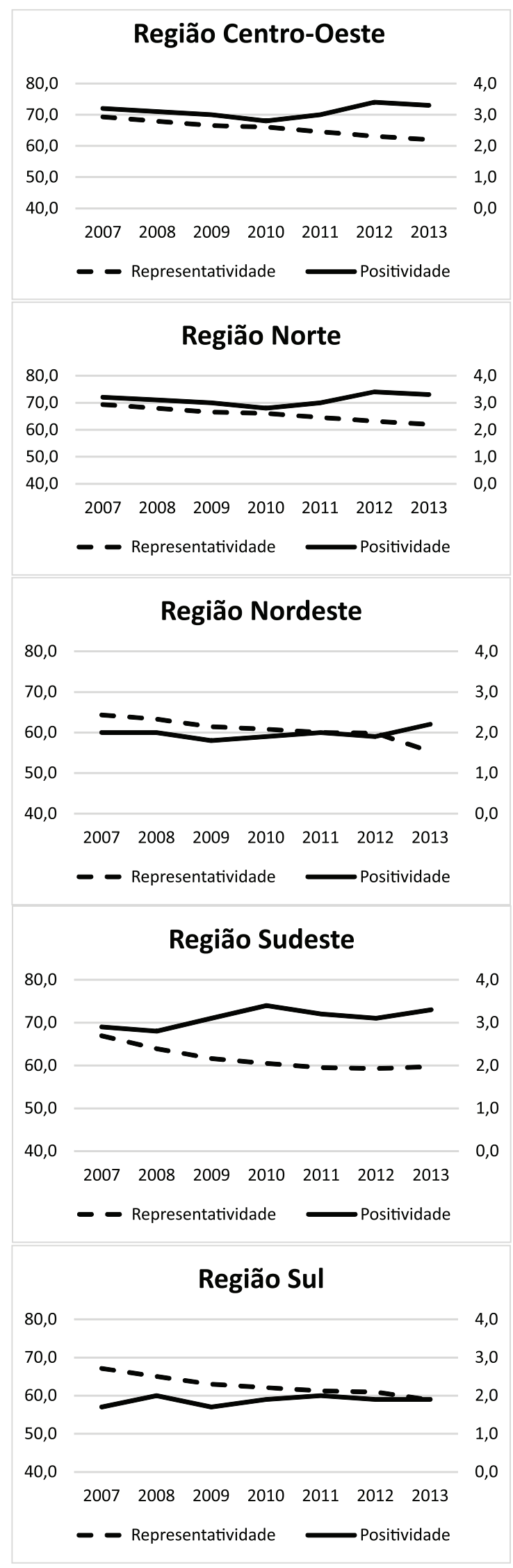

Figura 2. Representatividade da zona de transformação e positividade geral por Regiões, 2007-2013 
Sistema de Informação de Câncer (Siscan), sistema em plataforma web, em substituição ao Siscolo ${ }^{25}$.

A captação de mulheres na faixa etária alvo teve variaçáo positiva ao longo do período na maioria das Regióes, mas foi estacionária, mesmo em um grupo que estava sendo registrado pela primeira vez no Siscolo. Em análise realizada a partir dos exames registrados no Siscolo, entre 2006 e 2009, os autores observaram maior percentual de captação nas Regióes Norte, Nordeste e Centro-Oeste ${ }^{26}$. No estudo aqui apresentado, analisando mulheres, a Região Norte, apesar de tendência estacionária, foi a única com variação percentual negativa no período.

Um resultado inesperado é a positividade menor em mulheres captadas em relação à positividade geral, pois espera-se positividade maior em mulheres que fazem o exame pela primeira vez. Por outro lado, como a positividade geral é referente ao primeiro exame das mulheres registrados no Siscolo, é possível que falhas na informação da variável "fez preventivo anteriormente" tenham interferido no resultado, incluindo mulheres que já tinham feito o exame anteriormente entre as captadas, o que levaria a uma redução artificial da positividade no grupo.

Exames com representatividade da ZT apresentam maior chance de detecção de atipias celulares conforme identificado no estudo de Bastos et al. ${ }^{27}$, no qual, a partir dos dados do Siscolo no período de 2006 a 2009, observou-se que a chance de identificar lesōes foi cinco vezes maior entre exames com representatividade da ZT (odds ratio $=5,19)$ na faixa etária de 25 a 59 anos.

No presente estudo, a representatividade das amostras entre as mulheres examinadas apresentou tendência decrescente em todas as Regiōes e não foi observado o mesmo decréscimo em relação à positividade em algumas Regiōes. Uma possível explicação para esse resultado inesperado pode ser em virtude do aumento de idade ao longo do tempo, uma vez que mulheres mais velhas tendem a ter menor representatividade da ZT. A correlação entre representatividade da $\mathrm{ZT}$ e de positividade geral foi negativa e forte ou muito forte para quase todas as Regiōes. Para a Regiāo Sul, a partir de 2010, a correlação foi positiva e fraca, embora não estatisticamente significativa, provavelmente influenciada pela redução dos anos de observação.

Em 2013, em razão da implantação do Siscan ${ }^{25}$, o número de exames registrados no Siscolo teve uma importante queda em relaçáo ao ano anterior, principalmente nas Regióes Sudeste (cerca de um terço) e Sul (50\%). Por isso, os resultados de 2013 para o Brasil tiveram maior influência das Regiōes Norte, Nordeste e Centro-Oeste, o que pode ter provocado a mudança de padrão.

O rastreamento do câncer do colo do útero tem por objetivo identificar lesões precursoras que, se tratadas, não vão evoluir para o câncer e, assim, reduzir a incidência da doença. Nas Regiōes Sul, Sudeste e Nordeste, observou-se tendência de aumento na razáo entre lesão de alto grau e carcinoma epidermoide, indicando efetividade das ações de rastreamento.

Como possíveis limitaçóes do estudo, destaca-se o ponto de corte para iniciar o processo de limpeza da base de dados em 2007, quando foi implantada uma versão do sistema com a nova nomenclatura citopatológica, o que possivelmente levou à inclusão de algumas das mulheres que possuíam registro na versão anterior do sistema.

\section{CONCLUSÃO}

O estudo é relevante ao analisar os indicadores do programa por mulheres da população-alvo examinadas no SUS e indicar a tendência desses indicadores no país por grandes Regióes. A tendência temporal estacionária da captação e positividade em quase todas as Regiōes precisa ser acompanhada cuidadosamente, bem como a tendência decrescente da representatividade da ZT. Por outro lado, a razáo entre lesão de alto grau e câncer teve performance favorável, com tendência crescente para três Regiōes do país. Espera-se que os resultados contribuam para a discussão do controle do câncer do colo do útero, avançando de análises baseadas em exames realizados para mulheres examinadas por Estados e municípios de forma que açôes focais e locais possam auxiliar no controle dessa neoplasia.

\section{CONTRIBUIÇÕES}

Maria Beatriz Kneipp Dias, Vania Reis Girianelli, Arn Migowski, Caroline Madalena Ribeiro e Jeane Tomazelli contribuíram substancialmente na concepção e/ou planejamento do estudo; na obtenção, análise e/ou interpretação dos dados; na redação e revisão crítica. Luciana Leite de Mattos Alcântara contribuiu substancialmente na concepção e/ou planejamento do estudo; na obtenção, análise e/ou interpretação dos dados. Todos os autores aprovaram a versão final a ser publicada.

\section{DECLARAÇÃO DE CONFLITO DE INTERESSES}

Nada a declarar.

\section{FONTES DE FINANCIAMENTO}

Não há.

\section{REFERÊNCIAS}

1. Schiffman M, Wentzensen N. Human papillomavirus infection and the multistage carcinogenesis of 
cervical cancer. Cancer Epidemiol Biomarkers Prev. 2013;22(4):553-60. doi: https://doi.org/10.1158/10559965.EPI-12-1406

2. Instituto Nacional de Câncer José Alencar Gomes da Silva. Diretrizes brasileiras para o rastreamento do câncer do colo do útero. 2. ed. rev. atual. Rio de Janeiro: INCA; 2016.

3. World Health Organization. Cancer control: knowledge into action: WHO guide for effective programmes [Internet]. Geneva: World Health Organization; 2007 [cited 2020 Oct 23]. (Early Detection; module 3). Available from: http://www.who.int/cancer/modules/ Early\%20Detection\%20Module\%203.pdf

4. Instituto Nacional de Câncer José de Alencar Gomes da Silva. Sistemas de informação do controle do câncer de mama (SISMAMA) e do câncer do colo do útero (SISCOLO): manual gerencial. Rio de Janeiro: INCA; 2011.

5. Screening and Immunisations Team (NHS Digital); PHE, Screening (Public Health England). Cervical screening programme, England - 2019-20 [NS] [Internet]. England: NHS Digital; 2020 Nov 26 [cited 2020 Dec 10]. Available from: https://digital.nhs.uk/ data-and-information/publications/statistical/cervicalscreening-annual/england---2019-20

6. National Screening Unit [Internet]. Wellington, NZ: NSU; c2022. Cervical screening coverage; 2016 Apr 6 [cited 2020 Oct 23]. Available from: https://www.nsu. govt.nz/health-professionals/national-cervical-screeningprogramme/cervical-screening-coverage

7. Cervical cancer screening program 2015: annual report [Internet]. Vancouver, BC: BC Cancer Agency; 2015 [cited 2020 Nov 12]. Available from: http://www. bccancer.bc.ca/screening/Documents/CCSP_ReportAnnualReport2015.pdf

8. Performance monitoring for cervical cancer screening programs in Canada: report from the screening performance indicators working group, Cervical Cancer Prevention and Control Network (CCPCN) [Internet]. Ottawa: Public Health Agency of Canada; 2009 [cited 2020 Nov 12]. Available from: http://www.phac-aspc.gc.ca/cd-mc/cancer/ pmccspc-srpdccuc/pdf/cervical-eng.pdf

9. Public Health England NSP. NHS cervical screening programme: colposcopy and programme management [Internet]. London: NHS Cervical Screening Programme; 2016 [cited 2020 Nov 12]. Available from: https://www.bsccp.org.uk/assets/file/uploads/ resources/NHSCSP_20_Colposcopy_and_Programme_ Management_(3rd_Edition)_(2).pdf

10. Dias MBK, Tomazelli JG, Assis M. Rastreamento do câncer de colo do útero no Brasil: análise de dados do Siscolo no período de 2002 a 2006. Epidemiol Serv Saúde. 2010;19(3):293-306. doi: http://doi. org/10.5123/S1679-49742010000300011
11. Corrêa CSL, Lima AS, Leite ICG, et al. Rastreamento do câncer do colo do útero em Minas Gerais: avaliação a partir de dados do Sistema de Informaçáo do Câncer do Colo do Útero (SISCOLO). Cad Saúde Colet. 2017;25(3):315-23. doi: https://doi.org/10.1590/1414462X201700030201

12. Bortolon PC, Silva MAF, Côrrea FM, et al. Avaliaçáo da qualidade dos laboratórios de citopatologia do colo do útero no Brasil. Rev Bras Cancerol. 2012;58(3):43544. doi: https://doi.org/10.32635/2176-9745. RBC.2012v58n3.600

13. Magalhães JC, Morais LSF, Plewka J, et al. Evaluation of quality indicators of cervical cytopathology tests carried out in a municipality of Paraná, Brazil. J Bras Patol Med Lab. 2020;56:1-7. doi: https://doi.org/10.5935/16762444.20200041

14. Ribeiro CM, Santos Silva I, Eluf-Neto J, et al. Followup of women screened for cervical cancer in Sáo Paulo, Brazil: an analysis of the times to diagnostic investigation and treatment. Cancer Epidemiol. 2021;72:101940. doi: https://doi.org/10.1016/j.canep.2021.101940

15. Girianelli VR, Azevedo e Silva G, Thuler LCS. Factors associated with the risk of progression to precursor lesions or cervical cancer in women with negative cytologic findings. Int J Gynaecol Obstet. 2009;107(3):228-31. doi: https://doi.org/10.1016/j.ijgo.2009.07.036

16. Peres SV, Latorre MRDO, Michels FAS, et al. Determinação de um ponto de corte para a identificação de pares verdadeiros pelo método probabilístico de linkage de base de dados. Cad Saúde Colet. 2014;22(4):428-36. doi: https://doi.org/10.1590/1414462X201400040017

17. R Foundation for Statistical Computing. The R Project for Statistical Computing. [computer program]. R version 3.1.1. [Internet]. Vienna, AT: The R foundation; 2014. [cited 2020 Oct 20]. Available from: https:// www.R-project.org

18. Gasparin VA, Pitilin EB, Bedin R, et al. Fatores associados à representatividade da zona de transformaçáo em exames citopatológicos do colo uterino. Cogit Enferm. 2016;21(2):1-9. doi: http://doi.org/10.5380/ ce.v21i2.44241

19. Instituto Nacional de Câncer José de Alencar Gomes da Silva. Ficha técnica de indicadores das açôes de controle do câncer do colo do útero [Internet]. Rio de Janeiro: INCA; 2014 [acesso 2020 maio 10]. Disponível em: https://www.inca.gov.br/sites/ufu.sti.inca.local/files// media/document//fichatecnicaindicadorescolo14.pdf

20. Antunes JLF, Cardoso MRA. Uso da análise de séries temporais em estudos epidemiológicos. Epidemiol Serv Saúde. 2015;24(3):565-76. doi: https://doi.org/10.5123/ S1679-49742015000300024

21. Gujarati DN, Porter DC. Basic econometrics. 5th ed. Boston: McGraw-Hill Irwin; 2009. 
22. Callegari-Jacques SM. Bioestatística: princípios e aplicaçóes. Porto Alegre, SC: Artmed; 2009.

23. Ministério da Saúde (BR). Portaria no 1.504, de 23 de julho de 2013. Institui a Qualificação Nacional em Citopatologia na prevenção do câncer do colo do útero (QualiCito), no âmbito da Rede de Atenção à Saúde das Pessoas com Doenças Crônicas [Internet]. Diário Oficial da União. 2013 jul 24 [acesso 2020 out 23]; Seção I:31. Disponível em: https://bvsms.saude.gov.br/ bvs/saudelegis/gm/2013/prt1504_23_07_2013.html

24. Ministério da Saúde (BR), Secretaria de Vigilância em Saúde, Departamento de Análise de Situação de Saúde. Plano de açóes estratégicas para o enfrentamento das doenças crônicas não transmissíveis (DCNT) no Brasil, 2011-2022. Brasília, DF: Ministério da Saúde; 2011.

25. Ministério da Saúde. Portaria no 3.394, de 30 de dezembro de 2013. Institui o Sistema de Informação de Câncer (SISCAN) no âmbito do Sistema Único de Saúde (SUS) [Internet]. Diário Oficial da União. 2013 dez 31 [acesso 2020 out 23]; Seção I:57. Disponível em: http://bvsms.saude.gov.br/bvs/saudelegis/gm/2013/ prt3394_30_12_2013.html

26. Santos RS, Melo ECP, Santos KM. Análise espacial dos indicadores pactuados para o rastreamento do câncer do colo do útero no Brasil. Texto Contexto - Enferm. 2012;21(4):800-10. doi: https://doi.org/10.1590/ S0104-07072012000400010

27. Bastos EA, Zardo LMG, Feitosa TMP, Almeida RT. Associação entre a qualidade da amostra e a detecção de atipias celulares no exame citopatológico do colo do útero. Rev Bras Cancerol. 2012;58(3):445-52. doi: https://doi. org/10.32635/2176-9745.RBC.2012v58n3.601

Recebido em 10/2/2021

Aprovado em 11/5/2021 